\title{
Preservación de los microcentros históricos. Problemas de reuso entre conservación y compatibilidad
}

\author{
Maria Vitiello ${ }^{a}$ \\ a“Sapienza” Università di Roma, Dipartimento di Storia, Disegno e restauro dell'Architettura, Piazza Borghese, 9, 00186 Roma. \\ maria.vitiello@uniroma1.it
}

\begin{abstract}
Resumen
Las políticas de preservación de los centros históricos tienen raíces distantes. Desde la Carta de Venecia de 1964, la Declaración de Ámsterdam de 1975, hasta el documento firmado en Washington en 1987 y su revisión por el Comité CIVVIH del ICOMOS en 2011, la cultura de la restauración ha ampliado progresivamente los límites del patrimonio cultural que debe protegerse. Recientemente, los desafios en torno a la ciudad existente se han ido desvaneciendo gradualmente y el enfoque en los centros históricos ha ido perdiendo vigor, aunque su conservación se ha vuelto cada vez más problemática. Hoy en día se habla más a menudo de regeneración urbana, pero la restauración puede representar, más que en el pasado, la verdadera herramienta para el cuidado de los tejidos históricos, ya que esta disciplina basa el proyecto en un profundo conocimiento de los datos funcionales, estructurales y formales de cada realidad urbana sin excluir el entorno en todos sus aspectos. La conversión de muchos pequeños centros históricos abandonados en el interior de las montañas italianas en ciutad-hotel representa un sistema de valorización, pero no agota las necesidades conservadoras de estos núcleos urbanos. Al igual que el uso compatible no abarca todo el alcance del concepto de "conservación integrada". Esto, si se interpreta correctamente, podría, en cambio, representar la clave de la gestión del territorio mediante la planificación de un proceso de diseño en el que la restauración sea un medio de mediación entre las necesidades económicas, sociales y culturales de un sitio.
\end{abstract}

Palabras clave: pequeños centros históricos, regeneración urbana, conservación integrada.

\begin{abstract}
The preservation policies of historic centres have distant roots. From the Venice Charter of 1964, the Amsterdam Declaration of 1975, to the document signed in Washington in 1987 and its review by the ICOMOS CIVVIH Committee in 2011, the culture of restoration has progressively expanded the boundaries of the cultural heritage to be protected. Recently challenges around the existing city have gradually faded and the focus on historic centres has gradually lost vigour, although their conservation has become increasingly problematic. Today we hear more often about urban regeneration, but restoration can represent more than in the past, the real tool for the care of historical fabrics, as this discipline bases the project on a deep knowledge of the functional, structural and formal data of every urban reality without excluding the surrounding environment in all its aspects. The conversion of many small abandoned historical centres in the Italian mountain hinterland into widespread hotels represents a system of valorisation, but does not exhaust the conservative needs of these urban core. Just as compatible use does not cover the full breadth of the concept of "integrated conservation". This, if correctly interpreted, could, instead, represent the key to the management of the territory through the planning of a design process in which restoration is a means of mediation between the economic, social and cultural needs of a site.
\end{abstract}

Keywords: small historical centres, urban regeneration, integrated conservation. 


\section{Introducción. Centro histórico: definiciones y políticas de protección}

El Consejo de Europa declaró el año 1975 como el Año Europeo del Patrimonio Arquitectónico. Se llevaron a cabo muchas iniciativas para el conocimiento y la difusión de metodologías para el estudio del patrimonio construido en diferentes países. Los eventos, conferencias y seminarios se multiplicaron y la Carta Europea del Patrimonio Arquitectónico, adoptada por el Comité de Ministros del Consejo de Europa, es uno de los documentos que nos dejó esta serie de iniciativas. Es un documento de gran importancia porque amplía definitivamente la tutela del patrimonio arquitectónico desde la obra monumental, ejemplar por su singularidad, hasta la comprensión «de los conjuntos que conforman nuestras antiguas ciudades y nuestros pueblos tradicionales, en su entorno natural o construido». En ella se reconoce que «el patrimonio arquitectónico es un capital de valor espiritual, cultural, social y económico insustituible» y que sus condiciones requieren un enfoque coral para lograr una conservación integrada. En la Carta no se explica cómo puede hacerse explícita esa conservación (Jokilehto, 2011), pero queda claro en las palabras del texto: «La conservación es el resultado de la acción combinada de las técnicas de restauración y la búsqueda de funciones adecuadas. La evolución histórica ha llevado a que los centros degradados de la ciudad y, en ocasiones, las aldeas abandonadas se conviertan en reservas de viviendas de bajo costo». La Carta, ratificada en la conferencia final celebrada en los Países Bajos en octubre del mismo año e incorporada a la llamada Declaración de Amsterdam, es probable que sea el punto de acumulación de una serie de presiones dentro de la cultura de la restauración con respecto al cuidado del patrimonio arquitectónico en sentido amplio. Estos han estado presentes desde 1964, año en el que el Congreso Internacional de Arquitectos y Técnicos de Monumentos se reúne en Venecia del 25 al 31 de mayo. La declaración final firmada por los participantes se conoce como la Carta de Venecia de ICOMOS, en la que es posible encontrar una primera connotación amplia del concepto de patrimonio cultural, que llega a incluir «tanto la creación arquitectónica aislada como el entorno urbano o paisajístico que es el testimonio de una civilización particular, una evolución significativa o un acontecimiento histórico» (Carbonara, 1997; Vitiello, 2013). Los documentos posteriores a la Declaración de Ámsterdam se pueden reunir en dos grandes depósitos, para las dos cuestiones nodales que se abordan con respecto a la protección de los centros históricos. Por un lado, tenemos una producción bastante consistente de documentos a nivel internacional, que tienden a romper todos los límites posibles en la definición de patrimonio cultural, que se entiende cada vez más ampliamente, como lugar y paisaje (Carta de ICOMOS de Florencia en 1980 y de Burra en 1982) y a especificar las características de la conservación integrada (Carta de Cracovia de 2000). En el plano local, en Italia en particular, la legislación nacional trata de responder a una necesidad diferente puesta de relieve tanto por los documentos internacionales ya mencionados como por la Asociación Nacional de Centros Históricos y Artísticos, es decir, la necesidad de vincular estrechamente la planificación urbana y territorial a las cuestiones de conservación. La ley italiana $\mathrm{n}^{\circ} 457$ de 1978 introduce, de hecho, dentro de la legislación nacional el Plan de Recuperación, como instrumento de planificación urbana capaz de regular las intervenciones en las estructuras inmobiliarias existentes. Esta ley, si bien sólo aparentemente resuelve la cuestión del cuidado del patrimonio histórico de los edificios - de hecho, el art.31 «da legitimidad a muchas operaciones prohibidas por la cultura de la restauración» (Miarelli Mariani, 1992) - y deja abiertas las cuestiones relativas a los reglamentos técnicos y al espíritu empresarial, de hecho cierra un capítulo importante del debate italiano. En las décadas transcurridas desde los años ochenta, los retos culturales en torno a la ciudad histórica han abandonado por completo la vieja disputa antigo/nuevo, que había ocupado el debate en los años posteriores a la Segunda Guerra Mundial, avanzando tanto hacia la descripción de las formas necesarias para las prácticas de conservación de un patrimonio desplazado a contextos cada vez más amplios, como en el enfoque de los elementos que definen las características de los lugares, así como en la atención a las funciones sociales del patrimonio cultural. Actualmente la ciudad histórica, después de haber sido el terreno de viejos enfrentamientos entre renovadores y conservadores, entre arquitectos y urbanistas, entre restauradores e historiadores, entre administradores y técnicos, parece haberse extinguido - tal vez por cansancio o hambre - toda la tensión y las palabras que animaban esas antiguas disputas, que ahora parecen distantes y mudas, parecen haber perdido la fuerza para empezar de nuevo a defender esos lugares históricos de una erosión cultural, de una degradación tan profunda como opuesta (Musso, 2011). Por un lado, el corazón de las grandes ciudades se ha convertido en el lugar donde las enormes especulaciones económicas establecieron los flujos turísticos y modificaron el sistema de uso de los edificios, alaterando profundamente el tejido social, generando, en consecuencia, el fenómeno de la gentrificación. Por otra parte, los centros históricos de los pequeños núcleos urbanos, despojados de los servicios que la política insensata quiso 
concentrar en los grandes centros, muestran una degradación ambiental ligada sobre todo a la despoblación, a la decadencia social y, a veces, al abandono total.

Hoy, tal vez, deberíamos empezar de nuevo a pensar en el patrimonio cultural que representan los centros históricos de nuestras grandes y pequeñas ciudades, partiendo del análisis de los principales fenómenos de degradación de éstas, buscando soluciones dentro de los principios establecidos en la Carta Internacional de Protección de las Ciudades Históricas, promovida por el Consejo Internacional de ICOMOS en 1987 en Washington y, posiblemente, yendo más allá.

\section{La degradación, despoblación y abandono de los pequeños centros históricos}

Los magníficos pueblos medievales de origen antiguo que hacen nuestro paisaje único hoy en día están en condiciones de abandono, en una soledad desoladora. Transformados en verdaderas "ciudades fantasma", corren el riesgo de desaparecer, engullidos por el hambre del hombre y por una degradación cada vez más intensa, que borra irreversiblemente las huellas de un patrimonio histórico y cultural de inmensa belleza (Fig. 1). En estos pequeños centros urbanos, en los que la riqueza de la cultura arquitectónica italiana está muy extendida y es posible experimentar una integración armoniosa entre el patrimonio construido y el entorno natural, la degeneración causada por el abandono está cambiando profundamente las formas del edificio y está corroyendo esos lazos de memoria que los lugares son capaces de entretejer con las personas. En los últimos cincuenta años, Italia ha visto un cambio gradual pero radical en todos esos rasgos del paisaje que siempre han hecho a nuestro país único. Y esto ha ocurrido en un momento en el que casi el 75\% de la población ha elegido vivir en grandes ciudades (Di Biase, 2015). Esta situación se manifestó sobre todo en las zonas montañosas del interior de Italia, tanto en los Alpes como en los Apeninos. En estas regiones, desde la Segunda Guerra Mundial hasta hoy, se ha observado un fenómeno migratorio cíclico y bastante intenso, que ha afectado a diversos segmentos de la población.

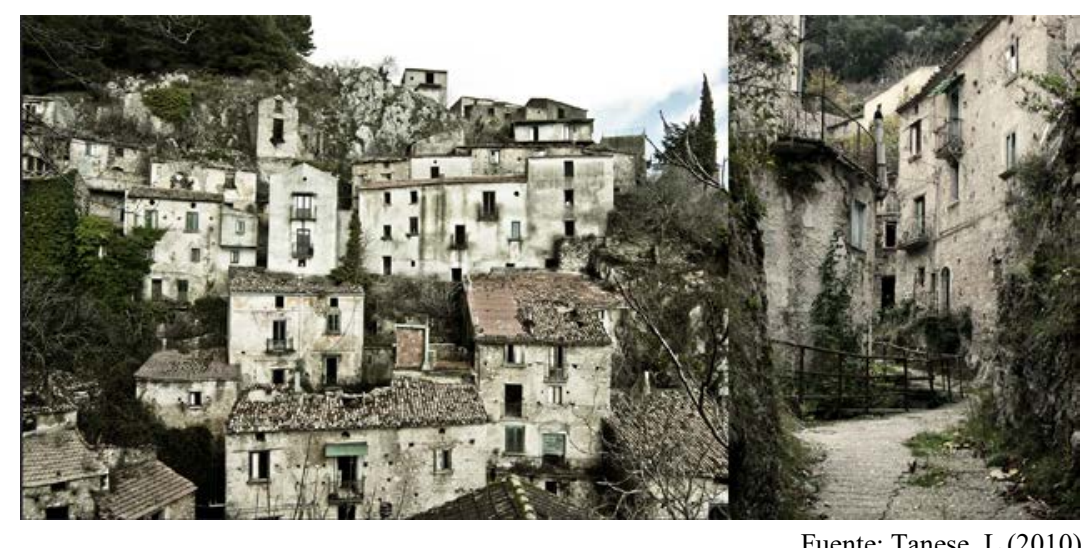

Fuente: Tanese, L (2010)

Fig. 1 Senerchia Vecchia. Un pueblo 'fantasma' en la provincia de Avellino. Italia

Las necesidades económicas, el reducido desarrollo industrial de estos lugares, la distancia de los principales enlaces de autopistas, las necesidades de vivienda cada vez más elevadas, el aislamiento interno de estos territorios junto con las repetidas catástrofes naturales debidas a los frecuentes y devastadores terremotos (como el que devastó L'Aquila y sus alrededores en 2009 o, más recientemente, el que involucró a las tierras de la Italia central en 2016-2017 que destruyó junto con Amatrice e Accumoli muchos pequeños centros históricos de los Apeninos centrales), puede ser considerada como las principales razones que han generado e intensificado este largo éxodo que aún impregna estas tierras. En efecto, en Italia hay regiones como Molise, el interior de Abruzzi, Marche, Lazio, Trentino-Alto Adige, Valle d'Aosta o las zonas más septentrionales de Piemonte que ven en sus territorios las numerosas pequeñas ciudades dispersas, antaño florecientes, transformadas en núcleos que no cuentan, en ocasiones, con más de 200 habitantes. De hecho, de los 7914 municipios italianos, sólo el 30,53\% tienen una población de más de 5.000 habitantes, mientras que 1953 tienen menos de 1.000 residentes y por útlimo hay 131 municipios con menos de 150 personas (ISTAT, 2019). En total, esta miríada de municipios que salpica el territorio nacional cubre un territorio que corresponde aproximadamente al $54 \%$ de la 
superficie total de Italia, sin embargo, alberga a menos del $20 \%$ de la población italiana, un porcentaje reducido a la mitad en comparación con los datos de hace un siglo (Fig. 2). Además de estos datos específicos y las consiguientes evaluaciones estadísticas o económicas relacionadas también con el mercado de la vivienda, a las que cabe remitirse para más detalles a estudios separados (Bevilacqua, 2012; ISTAT, 2019), puede afirmarse que esta "desertificación demográfica" que caracteriza a los territorios más interiores de Italia, que está relacionada con una acción política insensata incapaz de interceptar las necesidades de estas zonas y que hace recaer las razones de una inercia total sobre las características físicas del territorio, ha generado una intensa degradación física del entorno construido. Esta degradación pertenece tanto a la esfera del entorno construido como a la del espacio público y se refiere a la imposibilidad, incluso económica, de que el sector privado acceda a la intervención sobre el edificio mediante la predisposición de costosas obras de restauración, o al escaso interés de la administración pública por intervenir en la reurbanización de calles y plazas. De la confluencia de estos dos factores se derivan otras manifestaciones de degradación del sistema urbano, como la reducción progresiva de los servicios públicos y la sustitución de los habitantes tradicionales, reemplazados por nuevas personas sustancialmente ajenas a la estructura social original y pertenecientes, en cambio, a grupos sociales más débiles o incluso más fuertes, aunque atraídos por la oferta de viviendas de bajo costo. Esta diferencia no afecta al proceso de gentrificación, que siempre representa una sustitución progresiva del colectivo original por nuevos grupos sociales sustancialmente ajenos. Esta diferencia afecta, en cambio, al destino del propio centro, a la calidad de las intervenciones previstas en la edificación existente y, más en general, a la remodelación de los espacios públicos, así como a los servicios requeridos.

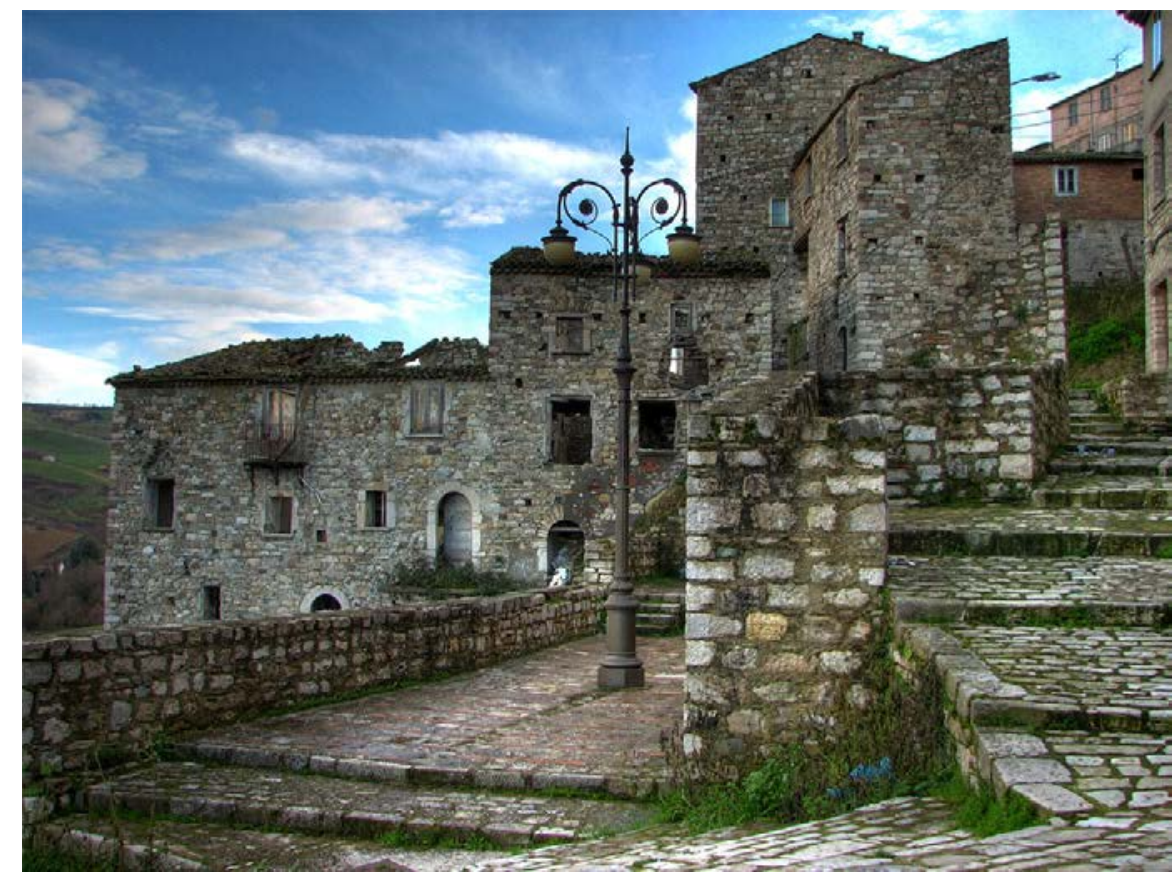

Fuente: Palladino, F (2010)

Fig. 2 Ripalimosani. Pequeña ciudad en el interior de Molise con el antiguo centro de la ciudad abandonado. Italia

Los centros históricos de nuestros núcleos urbanos son, por lo tanto, lugares frágiles y vulnerables; pero esta peculiaridad no puede hacernos olvidar las cualidades intrínsecas de los antiguos asentamientos, vinculadas a los sistemas arquitectónicos que los definen, a las articulaciones planovolumétricas tipológicas que los caracterizan, a los valores vinculados a la especificidad de los materiales utilizados en la edificación y a la configuración de los vacíos urbanos, a las calles de las plazas y a los vínculos que la morfología urbana establece con el territorio y el paisaje y a los valores sociales que este tipo de edificación y de configuración urbana consigue promover. Es en esta multiplicidad de valores, tangibles pero también intangibles, en todo caso todos plenamente trazables en la arquitectura como en los vacíos urbanos del antiguo palimpsesto, donde debe establecerse el enfoque de restauración más correcto y capaz de calibrar las intervenciones respetuosas de los muchos méritos del patrimonio construido histórico, con las necesidades modernas de la vida actual. 


\section{La restauración de la ciudad histórica, entre la reutilización y la regeneración}

Hoy en día, el tema de la preservación de los centros históricos parece haber pasado a diferentes niveles. De hecho, las cuestiones relativas al futuro de los edificios presentes en las cuencas urbanas preexistentes ya no se remiten a la restauración, ni a la recuperación o reutilización, incluso el debate "antiguo/nuevo" parece estar completamente superado por una «liquefazione» de la disciplina arquitectónica y por la autorreferencialidad de ciertas soluciones aplicadas concretamente a la existente. Hoy en día, los debates originales, en lugar de ser superados por los avances conceptuales, parecen más bien «anestetizzati» (Musso, 2011) y del silencio que ha caído sobre los temas de conservación de la ciudad existente y los pequeños centros históricos, nace una nueva perspectiva: la regeneración urbana. Se trata de una mutación sustancial y no meramente lingüística, en la que la diferencia léxica parece estar vinculada a una metodología operativa precisa, más que al azar de un sinónimo. Hoy en día, parece haber una necesidad urgente de cambiar el enfoque de las estrategias de estudio de la ciudad, así como de superar las disciplinas que tradicionalmente se han ocupado de la ciudad histórica. Así pues, se abandonan los conceptos tradicionales de planificación urbana y restauración de los siglos XIX y XX, en favor de la aceptación de instrumentos operativos y teóricos más capaces de interpretar la «realtà multistrumentali, che rendono la sfera architettonica inscindibile dalla politica organizzativa, urbana e delle nuove tecnologie» (Faroldi, 2015). La estrategia más efectiva parece ser la de la regeneración, para el territorio, para la ciudad, para la arquitectura. La regeneración urbana, por lo tanto, parece representar hoy en día el único sistema a través del cual se puede observar lo existente, con el fin de resolver las alteraciones ambientales, urbanas, sociales y arquitectónicas que la actividad antrópica ha generado a través de acciones frenéticas y temerarias en el antiguo tejido urbano. Pero para restablecer el elenco de valores de un centro histórico y la identidad que en él se coloca, ¿es realmente necesario establecer un nuevo proceso generativo dentro de la estructura preexistente? Lo cierto es que el escenario actual muestra una serie de problemas que requieren la superación de acciones simplistas, definidas dentro de campos estrechos y relaciones mecánicas entre disciplinas. Es evidente que es necesario ir más allá de los límites experimentados hasta ahora entre los proyectos arquitectónicos y urbanísticos, entre la composición y la tecnología como especialidades cerradas, hacia la aceptación de herramientas multiescala y multidimensionales. Resulta igualmente evidente que, en el momento en que se experimenta con las vías para la aplicación de la ciencia ecológica a las disciplinas relacionadas con la arquitectura, la extensión de los principios ecológicos a la ciudad existente es sólo un acto puramente consecuente. Pero la ecología «non è solo una disciplina capace di dare un'indicazione all'uomo su come comportarsi rispetto alla natura, ma è una scienza, esiste un complesso organico di conoscenze finalizzato allo studio delle relazioni tra gli organismi viventi e l'ambiente in cui sono legati» (Vitiello, 2012). En la definición de Arne Naess, la ecología se explica como «el estudio interdisciplinario de las condiciones de vida de los organismos en su interacción mutua con el medio ambiente circundante, tanto orgánico como inorgánico» (Naess, 1989). La ecología, de hecho, es el conocimiento científico y también un conocimiento descriptivo, que se puede resumir en el principio "all things hang together", que se puede traducir como: "todo depende de todo". Y las leyes fundamentales que la sustentan, que son las de la diversidad, la complejidad y la simbiosis, no son sólo descripciones rigurosas y lógico-matemáticas de las condiciones "objetivas" de la naturaleza, sino que son las mismas que sustentan la naturaleza compleja del patrimonio cultural y los mismos principios operativos de restauración. Esto se debe a que los sistemas ecológicos y sociales no son más que diferentes aspectos de una misma cuestión; una intuición que habían intuido los inventores de la encuesta social de finales del siglo XIX y que sin duda son las principales referencias a través de las cuales Roberto Pane llegó a comprender que la cuestión del medio ambiente para la disciplina de la restauración representa el «valor coral de la estratificación histórica» (Pane, 1959). Se trata de una interpretación que une el paisaje, la arquitectura, la naturaleza, la historia, la cultura filológica, la ciudad como palimpsesto, las necesidades psicológicas del hombre que la habita; un concepto que extiende su esfera de influencia sobre todo a las mallas del entorno construido en referencia a la ciudad antigua, pero que poco a poco llega a involucrar los problemas de lo que el propio Pane definirá, unos años más tarde, como "ecología humana". La disciplina de la restauración, en otras palabras, se combina de manera práctica y conceptual con la de la ecología, con la que comparte tanto el objeto de estudio, es decir, el patrimonio cultural entendido en su más amplia extensión, como el lugar, tal como se definió en Burra en 1982, y el hombre que utiliza y goza de sus beneficios, así como la metodología de acercamiento al análisis de lo existente realizada con vistas a su transmisión al futuro. Por lo tanto, incluso antes de pensar en nuevas estrategias regenerativas, sería necesario comenzar de nuevo a estudiar los principios de restauración establecidos por la Carta 
Internacional para la Preservación de las Ciudades Históricas, promovida por el Consejo Internacional del ICOMOS en 1987 en Washington, y sólo más tarde comprender cómo superarlos.

\subsection{La Carta del ICOMOS de 1987 y su actualización}

La relectura de la Carta de Washington y la actualización realizada por el Comité Internacional de ICOMOS CIVVIH en La Valletta, ratificada por la Asamblea General celebrada en París en 2011, permite centrar la atención en algunos temas importantes que van más allá de la definición del centro histórico como patrimonio cultural que aparece en la introducción del Documento de Venecia de 1964 y que, de hecho, se basa en la definición coral del patrimonio cultural como «una creación arquitectónica aislada y un entorno urbano o paisajístico que constituye el testimonio de una civilización particular, una evolución significativa o un acontecimiento histórico», que representa «un capital espiritual, cultural, económico y social de valor irreemplazable», como añade la Declaración de Ámsterdam (Carbonara, 1997).

El objetivo principal de los documentos mencionados está claramente establecido en la calificación metodológica de la acción de salvaguardia de las ciudades y los barrios históricos. En estos documentos, el conocimiento del sitio se considera un acto protagonista en la evaluación de cada proyecto para una conservación y mejora consciente de los asentamientos urbanos existentes. Se recuerdan los valores fundamentales que pueden reconocerse en los entornos urbanos históricos y que, por lo tanto, están en la base de la conservación de un lugar: el carácter procedimental del paisaje urbano, ofrecido también como un conjunto de todos los elementos materiales e inmateriales, vinculado a la integridad visual de la imagen conectada con la identidad colectiva de un lugar.Estos componentes son detectables por las cualidades paisajísticas e identitarias del asentamiento histórico que, específicamente, están dadas por "la forma urbana, que se define por la red de calles y la subdivisión de las zonas urbanas; las relaciones entre los diferentes espacios urbanos: espacios construidos, espacios abiertos, espacios verdes; la forma y el aspecto de los edificios (internos y externos), definidos por su estructura, volumen, estilo, escala, material, color y decoración; las relaciones de la ciudad con su entorno natural o artificial, las diferentes vocaciones de la ciudad adquiridas a lo largo de su historia» (ICOMOS 1987). La metodología interpretativa sugerida por la Carta, y su posterior actualización, es por lo tanto multiescalar porque incluye los diversos componentes del medio ambiente: desde el territorio con sus factores paisajísticos, al asentamiento urbano, al tejido edilicio, a los vacíos urbanos, a la comprensión de la calidad de los espacios verdes, la caracterización de la arquitectura y las técnicas de construcción. Obviamente, esta recopilación de datos no puede ser exhaustiva en sí misma, sino que necesita una lectura sistémica, capaz de interconectar las diferentes escalas del sitio y no puede agotarse ni siquiera en la mera comprensión de las transformaciones antrópicas del sitio. El diseño para la conservación de las ciudades históricas requiere estudios interdisciplinarios que, además de los datos arqueológicos, arquitectónicos y técnicos, deben incluir aspectos sociológicos y económicos, con el fin de definir acciones legales, administrativas y financieras dentro del proyecto de restauración. La disputa "antiguo/nuevo", en torno a la cual el debate sobre los temas de la restauración a escala urbana, al menos en Italia, ha estado oscilando durante algún tiempo, parece superada definitivamente por el concepto de "conservación integrada" que admite, en principio, el derecho del restaurador a intervenir "sobre" - o "en" - lo construido a través de los instrumentos lingüísticos y tecnológicos de hoy en día, pero sobre todo implica un entrelazamiento muy estrecho de conocimientos, planificación y normas. Evidentemente, no podemos dejar de subrayar aquí el papel central del conocimiento, que no puede ser una mera acumulación de información, sino que debe representar un análisis preciso de lo existente, aunque sea en la intersectorialidad de la información y en la capacidad del proyecto de sistematizar las diferentes áreas del conocimiento y en la confrontación abierta con la población donde se pone de manifiesto la "integración" y la "participación" que evocan repetidamente los Documentos. Por lo tanto, esta comparación debe leerse más allá de «el resultado del uso combinado de técnicas de restauración y la búsqueda de funciones apropiadas» (De Fusco, 1992). La reducción de este complejo concepto a un análisis de compatibilidad de uso o reutilización no puede ser compartida, ni tampoco puede aceptarse que se pueda leer como la inclusión de la arquitectura contemporánea en los barrios antiguos. Pero lo que debe abordarse en las Cartas, que también faltan en la Recomendación de la UNESCO sobre el Pesaje Urbano Histórico (París 2011), es el tema de la continuidad de uso, que es un tema central para el estudio y la restauración de los pequeños centros históricos. Éstos no sufren, de hecho, los problemas generados por el tráfico, el turismo o la superpoblación en general, sino que padecen la soledad y el abandono. Y éste es un tema que se ha abordado, al menos 
hasta ahora, de manera muy superficial, mientras que se han puesto de relieve los problemas de los grandes centros urbanos, ricos en arte, naturaleza y paisaje que hay que salvaguardar.

\subsection{Aspectos de la reutilización de los pequeños centros históricos}

El tema de la reutilización, sin embargo, parece ser un nudo importante pendiente de desatar, especialmente en el caso de los pequeños centros históricos, ya que el tema de su rehabilitación se está convirtiendo en un campo de interés muy importante, debido a las oportunidades sociales y económicas relacionadas con ellos y sobre las que se tiende a «fundamentar una nueva idea de conservación y valorización, produciendo valor añadido, crecimiento y renacimiento en contextos degradados y/o afectados por fenómenos de abandono» (Villani y Dall'ara, 2015). Las estrategias de intervención experimentadas hasta ahora en este patrimonio arquitectónico difundido en el terrriotrio interno de las regiones montañosas italianas, tienen por objeto especificar los fines turísticos, que parecen capaces, más que otros, de salvaguardar el valor de identidad de los sitios, sin dejar de lado los procesos de cambio indisociables de cualquier tipo de valorización del patrimonio que se vaya a realizar.

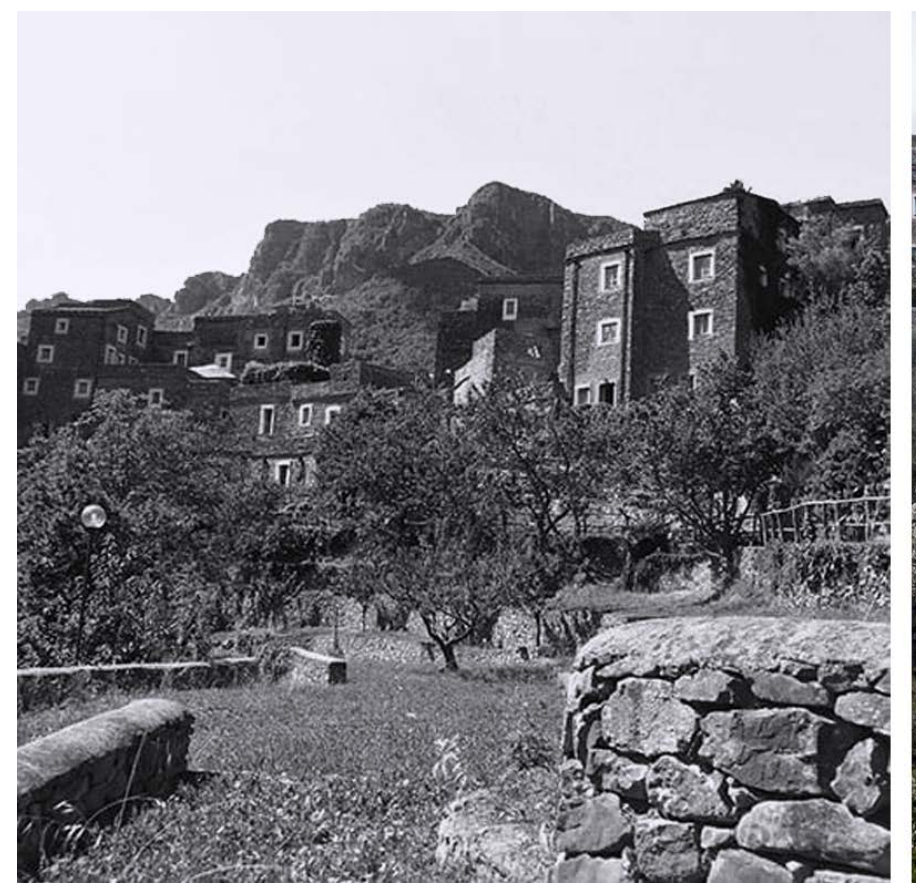

Fig. 3 Colletta di Castelbianco. Savona. Restauración urbana por Giancarlo De Carlo en 1990

Evidentemente se trata de una reutilización sostenible, por lo tanto también de un turismo sostenible, es decir, un turismo montado sobre estructuras de alojamiento "horizontales", en el que la hospitalidad se vierte sobre toda la zona urbana histórica y el uso de la edificación se modula en función de una cuidadosa evaluación del cambio para cada arquitectura, ya sea un elemento destacado o una celda del tejido edilicio básico del asentamiento urbano. Actualmente el llamado "Albergo Difuso" es un tipo de alojamiento turístico planificado y regulado localmente por todas las regiones italianas y los ejemplos realizados en nuestro país son muy numerosos (Villani y Dall’Ara, 2015). Se trata de realizaciones muy significativas promovidas cuidadosamente por la Asociación Internacional de Hoteles Difusos, cuyo principal compromiso es promover el conocimiento de un modelo de alojamiento completamente innovador que se basa en un turismo cultural no masivo, que tiende a revivir los lugares más que a consumir su identidad (Fig. 3). Ciertamente, las experiencias realizadas en este sentido en casi veinte años desde los primeros experimentos constituyen una excelente base para la formulación de un primer balance, del cual no puede sino emerger que el verdadero éxito del modelo según los objetivos de regeneración prefigurados al principio (Faroldi, 2015). La efícacia del sistema económico, junto con la planificación de una restauración verdaderamente conservadora tanto a nivel del sistema arquitectónico como a nivel de la estructura urbana y social, ha generado una serie de efectos positivos en los territorios circundantes. 


\section{Conclusiones}

Aunque los resultados realmente positivos generados por estos nuevos usos de la edificación en los pequeños centros históricos pueden contribuir a definir el "Albergo Diffuso", ciudad-hotel, como un verdadero "motor" para la reurbanización del patrimonio urbano histórico en comparación con la plena compatibilidad de las nuevas funciones, es difícil imaginar un denominador común en este tipo de uso para la miríada de centros históricos despoblados o completamente abandonados del territorio. La estrategia de conservación que se ensaye para estos pequeños centros urbanos debería centrarse realmente en la conservación integrada, en la que no sólo se considere la rehabilitación de los edificios existentes en relación con el uso turístico, sino que debería enmarcarse en un espacio de diseño más amplio. Cabe recordar aquí, que el principal postulado de la Carta Europea del Patrimonio Arquitectónico ratificada en Amsterdam en 1975, la conservación del patrimonio arquitectónico de un sitio depende "en gran medida de su integración en la vida de sus ciudadanos y de su función en la planificación urbana y regional". Por lo tanto, no es en la identificación de un uso compatible donde se disuelve la complejidad del concepto de conservación integrada. Esto representa un elemento fundamental para la recuperación conservadora de las arquitecturas individuales (Miarelli Mariani, 1992), pero es en la dimensión territorial del diseño donde debe encontrarse la verdadera integración. Sin embargo, para ello es necesario enmarcar la cuestión de su conservación sobre una base más amplia, en el gobierno del territorio. Es necesario actuar sobre las políticas públicas, como en algunos aspectos ya sugeridos por el documento de la UNESCO mencionado anteriormente, pero no tanto para la protección de las posibles transformaciones del patrimonio cultural existente, sino para las políticas sostenibles de reequilibrio social y urbano y para evitar un mayor consumo del territorio. En otras palabras, para ser verdaderamente efectivos, nuestro trabajo en los pequeños centros históricos necesita un diseño programático amplio, interdisciplinar, sabiamente definido y compartido. Esto debería llevarse a cabo mediante un proceso integrado, "capaz de cruzar las necesidades económicas, sociales y culturales y mantener un control constante de los efectos en todos los campos" (Gabrielli, 2011), incluyendo proyectos de restauración en este proceso. Todo ello para que el paisaje cultural fruto de la articulación de los pequeños centros históricos, pueda ser verdaderamente salvaguardado.

\section{Referencias}

Bevilacqua, P. (2012). Precedenti storici e caratteristiche del declino delle aree interne. En Le aree interne: nuove strategie per la programmazione 2014-2020 della politica di coesione territoriale, Congreso desarrollado en Roma, 15 de diciembre de 2012. Recuperado de http://www.dps.tesoro.it/Aree interne/seminario 1212.asp

Carbonara, G. (1997). Avvicinamento al Restauro. Nápoles: Liguori Editore.

De Fusco, R. (1992). Dentro e fuori l'architettura: scritti brevi (1960-1990). Milán: Jaka Book.

Di Biase, C. (2015). Small town in inland areas: shared landscape. En R. Crisan, D. Fiorani, L. Kealy, y S. Musso (Eds.), Conservation reconstruction. Small Historic Centres. Conservation in the midst of change (pp. 131-140). Hasselt: AEEA.

Faroldi, E. (2015). Strategie regenerative per il territorio, la città, l'architettura. Techne, 10, 6-10

Gabrielli, B. (2011). 50 anni di ANCSA: le idee di ieri, le responsabilità dell'oggi. En F. Toppetti (Ed.), Paesaggi e città storica. Teorie e policiche del progetto (pp. 9-18). Florencia: Alinea Editrice.

Jokilehto, J. (2011). L'evoluzione della dottrina internazionale. En F. Toppetti (Ed.), Paesaggi e città storica. Teorie e policiche del progetto (pp. 115-126). Florencia: Alinea Editrice.

Miarelli Mariani, G. (1992). Centri storici. Note sul tema. Roma: Bonsignori Editore.

Musso, S. (2011). Per un insegnamento integrato del progetto della città esistente. En F. Toppetti (Ed.), Paesaggi e città storica. Teorie e policiche del progetto (pp. 147-151). Florencia: Alinea Editrice.

Naess, A. (1989). Ecology, Community and Lifestyle. Cambridge: Cambridge University Press.

Pane, R. (1959). Città antiche edilizia nuova. Nápoles: ESI.

Villani, T., Dall'Ara, G. (2015). L'albergo diffuso come modello di sopitalità originale e di sviluppo dei borghi. Techne, 10, $170-177$.

Vitiello, M. (2013). Prospettive ecologiche per il restauro. Milán: Franco Angeli. 